\title{
Correction to: Giant nonfunctioning adrenal tumors: two case reports and review of the literature
}

George Chatzoulis, Ioannis Passos*, Dimitra-Rafailia Bakaloudi, Dimitrios Giannakidis, Alexandros Koumpoulas, Konstantinos Ioannidis, Ioannis Tsifountoudis, Dimitrios Pappas and Panagiotis Spyridopoulos

\section{Correction to: J Med Case Reports (2018) 12:335}

https://doi.org/10.1186/s13256-018-1876-8

In the publication of this article [1], there is an error in the Family Name and Given Name of the authors since these were interchanged.

\section{The error:}

Chatzoulis George, Passos Ioannis", Bakaloudi DimitraRafailia, Giannakidis Dimitrios, Koumpoulas Alexandros, Ioannidis Konstantinos, Tsifountoudis Ioannis, Pappas Dimitrios and Spyridopoulos Panagiotis

Should instead read:

George Chatzoulis, Ioannis Passos", Dimitra-Rafailia Bakaloudi, Dimitrios Giannakidis, Alexandros Koumpoulas, Konstantinos Ioannidis, Ioannis Tsifountoudis, Dimitrios Pappas and Panagiotis Spyridopoulos

This has now been updated in the original article [1].

Received: 15 November 2018 Accepted: 15 November 2018

Published online: 06 December 2018

\section{Reference}

1. Chatzoulis G, Passos I, Bakaloudi D-R, et al. Giant nonfunctioning adrenal

tumors: two case reports and review of the literature. J Med Case Reports. 2018;12:335. https://doi.org/10.1186/s13256-018-1876-8

\footnotetext{
* Correspondence: ioannispassos@gmail.com

Department of Surgery, 424 General Military Hospital of Thessaloniki, Agiou Nikolaou 42, 55,132, Kalamaria, Thessaloniki, Greece
} 